UDC 554.47:547.514.7

\title{
THE KINETICS AND MECHANISM OF THE SELECTIVE OXIDATIVE DEHYDROGENATION REACTION OF METHYLCYCLOPENTANE
}

\author{
A.M.Aliyev, M.Y.Abbasov, M.G.Aliyeva, G.A.Alizade, R.Yu.Agayeva \\ M.Nagiyev Institute of Catalysis and Inorganic Chemistry, NAS of Azerbaijan \\ abbasov.mahir@mail.ru
}

Received 20.04.2021

Accepted 27.05.2021

\begin{abstract}
The oxidative dehydrogenation of alicyclic diene hydrocarbons refers to scarcely studied heterogeneous catalytic reactions which proceed with the participation of oxygen. The dehydrogenation of methylcyclopentane is an endothermic reaction. To improve the reaction kinetics, this research was to develop a structured catalyst by conductive metals $(\mathrm{Cu}, \mathrm{Zn}, \mathrm{Co}, \mathrm{Cr})$ support which could hold an adherent catalytic layer. The active phase was impregnated onto these support metals and the developed catalyst was tested for the dehydrogenation of methylcyclopentane. The catalyst preparation involved three main key steps which were support oxidative reaction, loading of active particles on the catalyst surface, preparation of an active catalyst layer on the surface finally bringing the catalyst into the active phase. Different types of catalyst activation and deactivation mechanisms stability have been studied in this investigation. The advantage of this works, the oxidative dehydrogenation of methylcyclopentane is that it occurs at the expense of oxygen in the air. The zeolite structure study helped identify the effect of the combination of catalysts, and adsorption of metals on clinoptilolite and dispersion on the selectivity of the catalyst particles. Numerical values of the kinetic parameters were calculated.
\end{abstract}

Keywords: kinetics, oxidative dehydrogenation, methylcyclopentadiene-1,3.

doi

\section{Introduction}

The alicyclic dienic hydrocarbons are the raw material for the synthesis of various classes of polyfunctional compounds. Functional derivatives of these compounds by reason of the high activity of a multiple bond are used in the synthesis of polymeric and composite materials for special purposes, physiologically active compounds, as well as the chiral synthons for the directed obtaining analogues of natural compounds and medicaments. The most widely studied reaction of catalytic dehydrogenation of naphthenes hydrocarbons in the oil field $[1,2]$. It is known that this thermodynamically limited reaction proceeds under hard conditions lead to aromatization and resinification of significant part of the hydrocarbon fraction, as well as rapid coking and deactivation of the catalysts. This type of oxidative dehydrogenation of methylcyclopentan for obtaining of methylcyclopentadiene-1,3 refers to poorly studied heterogeneous catalytic reactions proceed with participation of oxygen. The using of molecular oxygen as an acceptor of hydrogen allows one to carry out the process at milder conditions and to prevent the above mentioned difficulties [3]. This invention relates to a novel catalyst and to the use of this catalyst in the cata- lytic dehydrogenation of methylcyclopentane to produce methyl-1,3-cyclopentadiene. Throughout the specifications and claims of this application methylcyclopentadiene-1,3 is used generically to encompass all positional isomers as determined by the position of the methyl group on the cyclopentadiene ring. Methylcyclopentadiene-1,3 is an extremely useful compound for preparation of intermediates synthetic resinous compositions [4]. In this case, the synthesis of highly active and selective zeolite-based catalyst for the selective oxidative dehydrogenation of methylcyclopentane to methylcyclopentadiene1,3 is of great theoretical and practical importance. This investigation present the results of studies of oxidative dehydrogenation of methylcyclopentane with molecular oxygen in order to identify the conditions of selective formation of methylcyclopentadiene-1,3 in the presence of modified zeolite catalysts and kinetics results of the reaction.

\section{Experimental part}

Catalysts prepared by the ion-exchange method, using Azerbaijan natural zeolites clinoptilolite (crystallinity $-84.0 \%, \lambda=8.68$ ) modified by various cations, transition and nontransition elements $(\mathrm{Zn}, \mathrm{Cu}, \mathrm{Co}, \mathrm{Cr}, \mathrm{Mn}, \mathrm{Fe}$, 
etc.). Zeolites $\mathrm{NaY}\left(\mathrm{SiO}_{2} / \mathrm{Al}_{2} \mathrm{O}_{3}, \lambda=4.2\right), \mathrm{NaX}$ $(\lambda=2.9), \mathrm{NaA}(\lambda=2.0)$ and mordenite $(\lambda=9.6)$. Modification of zeolites with metal cations was performed by treatment their initial forms in a solution of chloride salts of the corresponding cations. The catalysts prepared on the basis of clinoptilolite and mordenite were subjected to acid treatment before the ion exchange. Firstly the natural zeolites have been treated with $0.5 \mathrm{~N}$ $\mathrm{HCl}$. Number of the cations incorporated into the zeolite was determined by ICP-MS Agilent 7700 and amounted to $0.1-2 \%$ by mass of the zeolite. Catalysts particles size $0.25-0.63 \mathrm{~mm}$ and purity of methylcyclopentane were $99.5 \%$ in this work. The reaction is carried out in a U-shaped flow reactor. The reactor is placed in a air electric oven with automatic temperature control. Feed of methylcyclopentane was carried out by a pump. Methylcyclopentane vapors and nitrogen mixed with oxygen in the mixer located in a thermostated oven and enter the reactor with the catalyst. Temperature stability is maintained in the oven with a contact thermometer. The temperature in the middle of the catalyst bed is measured with a thermocouple and recorded with the potentiometer. The reaction unit is directly connected to the analysis system through six-way valve allows the analysis of the reaction mixture to be without loss. The mixture exiting from the reactor gradually passes through the sample loop and is collected in cooled trap. The analyses of the product of the reactions were also performed by gas chromatography using GC "Agilent 7890" with "Agilent 5975" MS and capillary column HP-5MS (length-30 m).

\section{Results and Discussion}

Discovered of that, the conversion of methylcyclopentane over studied catalysts occurs in the following directions: oxidative dehydrogenation; partial and deep oxidation. The yield of reaction products depend from the porous structure of the zeolite, its physico-chemical properties as well as the nature and concentration of cations incorporated in the zeolites and reaction conditions. Initially has been investigated catalytic activity of the initial zeolites in oxidative dehydrogenation of methylcyclopentane. The key differ- ence between natural heulandite and clinoptilolite is thermal stability. Clinoptilolite can also having alkali cations dominant, $[(\mathrm{Na}+\mathrm{K})>\mathrm{Ca}]$ [5]. Clinoptilolite can be represented by typical oxide formula as: $\left(\mathrm{Na}_{2}, \mathrm{~K}_{2}\right) \mathrm{O} \cdot \mathrm{Al}_{2} \mathrm{O}_{3} \cdot 10 \quad \mathrm{SiO}_{2} \cdot 8$ $\mathrm{H}_{2} \mathrm{O}$. Unit cell contents of clinoptilolite can be represented as $(\mathrm{Na}, \mathrm{K})_{6} \cdot\left(\mathrm{Al}_{6} \quad \mathrm{Si}_{30} \mathrm{O}_{72}\right) \cdot 24 \quad \mathrm{H}_{2} \mathrm{O}$. The unit cell of clinoptilolite is monoclinic and is usually characterized on the basis of 72 oxygen atoms $(n=36)$ and $m=24$ water molecules, with $\mathrm{Na}^{+}, \mathrm{K}^{+}, \mathrm{Ca}^{2+}$ and $\mathrm{Mg}^{2+}$ as the most common charge-balancing cations [6]. This is more likely due to the strong adsorption of methylcyclopentane on faujasite. In contrast to zeolites with large pores $(\mathrm{NaX}, \mathrm{NaY})$, narrow porous zeolites characterized with minor surface $(8.0-20.0) \mathrm{m}^{2} / \mathrm{g}$ and small pore size $(4.2-4.9) \AA$ promote the selective conversion of methylcyclopentane to methylcyclopentene.

Kinetic experiments were performed in a flow apparatus at atmospheric pressure in the temperature range $(320-360)^{0} \mathrm{C}$, space velocity $500-2000 \mathrm{~h}^{-1}$, the partial pressures of the reactants $\mathrm{C}_{6} \mathrm{H}_{12} P=0.22-0.32$ atm; $\mathrm{O}_{2} P=0.14$ $0.17 \mathrm{~atm}$. Runs performed at several feed rates and using granules of the catalyst $\mathrm{Cu} \mathrm{Zn} \mathrm{Co} \mathrm{Cr}$ clinoptilolite with of different sizes showed that external and internal mass transfer effects were negligible under the studied experimental conditions. The mechanism of oxidative dehydrogenation methylcyclopentane on above mentioned active centers may be presented as following stage scheme:

$$
\begin{aligned}
& \mathrm{O}_{2}+2 \mathrm{Z}_{1} \stackrel{k_{1}}{\longrightarrow} 2 \mathrm{Z}_{1} \mathrm{O}|1 / 2| \\
& \mathrm{O}_{2}+2 \mathrm{Z}_{2} \stackrel{k_{2}}{\longrightarrow} 2 \mathrm{Z}_{2} \mathrm{O}|1 / 2| \\
& \left(\mathrm{Z}_{1} \mathrm{O}+\mathrm{Z}_{2} \mathrm{O}\right)+\mathrm{C}_{6} \mathrm{H}_{12} \stackrel{k_{3}}{\longrightarrow}\left(\mathrm{Z}_{1} \mathrm{O}+\mathrm{Z}_{2} \mathrm{O}\right) \mathrm{C}_{6} \mathrm{H}_{12}|1| \\
& \left(\mathrm{Z}_{1} \mathrm{O}+\mathrm{Z}_{2} \mathrm{O}\right) \mathrm{C}_{6} \mathrm{H}_{12} \stackrel{k_{4}}{\longrightarrow} \mathrm{C}_{6} \mathrm{H}_{8}+2 \mathrm{H}_{2} \mathrm{O}+\mathrm{Z}_{1}+\mathrm{Z}_{2}|1| \\
& \mathrm{C}_{6} \mathrm{H}_{12}+\mathrm{O}_{2}=\mathrm{C}_{6} \mathrm{H}_{8}+2 \mathrm{H}_{2} \mathrm{O}
\end{aligned}
$$

The following expression can be written for rates of the stages:

$$
\begin{aligned}
& r_{1}=k_{1} \theta_{1}^{2} P_{\mathrm{O}_{2}}, r_{2}=k_{2} \theta_{2}^{2} P_{\mathrm{O}_{2}}, r_{3}=k_{3} \theta_{3} P_{\mathrm{C}_{6} \mathrm{H}_{12}}, \\
& r_{4}=k_{4} \theta_{4}
\end{aligned}
$$

In stationary conditions

$r=r_{1}=r_{2}=r_{3}=r_{4}$. 
Here $\theta_{1}, \theta_{2}, \theta_{3}$, and $\theta_{4}$ are fractions of catalyst surface; the sites with two near arranged adsorbed atom oxygen on different exchange cations and the sites with adsorbed molecules of methylcyclopentane.

$$
\begin{aligned}
& \theta_{4}=\frac{k_{3} \theta_{3} P_{\mathrm{C}_{6} \mathrm{H}_{12}}}{k_{4}} \quad \theta_{1}=\sqrt{\frac{k_{3} P_{\mathrm{C}_{6} \mathrm{H}_{12}}}{k_{1} P_{\mathrm{O}_{2}}}} \cdot \sqrt{\theta_{3}}, \\
& \theta_{2}=\sqrt{\frac{k_{3} P_{\mathrm{C}_{6} \mathrm{H}_{12}}}{k_{2} P_{\mathrm{O}_{2}}}} \cdot \sqrt{\theta_{3}}, \theta_{1}+\theta_{2}+\theta_{3}+\theta_{4}=1
\end{aligned}
$$$$
\frac{k_{3} P_{\mathrm{C}_{6} \mathrm{H}_{12}}}{k_{4}} \theta_{3}+\theta_{3}+\left(\sqrt{\frac{k_{3} P_{\mathrm{C}_{6} \mathrm{H}_{12}}}{k_{1} P_{\mathrm{O}_{2}}}}+\sqrt{\frac{k_{3} P_{\mathrm{C}_{6} \mathrm{H}_{12}}}{k_{2} P_{\mathrm{O}_{2}}}}\right) \times
$$$$
\times \sqrt{\theta_{3}}-1=0
$$

Assuming

$$
\begin{gathered}
\frac{k_{3} P_{\mathrm{C}_{6} \mathrm{H}_{12}}}{k_{4}}=A, \quad\left(\sqrt{\frac{k_{3} P_{\mathrm{C}_{6} \mathrm{H}_{12}}}{k_{1} P_{\mathrm{O}_{2}}}}+\sqrt{\frac{k_{3} P_{\mathrm{C}_{6} \mathrm{H}_{12}}}{k_{2} P_{\mathrm{O}_{2}}}}\right)=B \\
\theta_{3}=x^{2}, \sqrt{\theta_{3}}=x, \quad A x^{2}+B x-1=0
\end{gathered}
$$

We'll receive:

$$
\begin{aligned}
& \frac{-B+\sqrt{B^{2}+4 A}}{2 A} \\
& \theta_{3}=x^{2}=\left(\frac{-B+\sqrt{B^{2}+4 A}}{2 A}\right)^{2}
\end{aligned}
$$

Then the equation of rate of the formation methylcyclopentadiene appears as

$$
r=r_{\mathrm{C}_{6} \mathrm{H}_{8}}^{1}=k_{3} P_{\mathrm{C}_{6} \mathrm{H}_{12}} \cdot\left(\frac{-B+\sqrt{B^{2}+4 A}}{2 A}\right)^{2}
$$

$$
r=r_{\mathrm{C}_{6} \mathrm{H}_{8}}^{1}=k_{3} P_{\mathrm{C}_{6} \mathrm{H}_{12}} \cdot\left(\frac{\left.-\left(\sqrt{\frac{k_{3} P_{\mathrm{C}_{6} \mathrm{H}_{12}}}{k_{1} P_{\mathrm{O}_{2}}}}+\sqrt{\frac{k_{3} P_{\mathrm{C}_{6} \mathrm{H}_{12}}}{k_{2} P_{\mathrm{O}_{2}}}}\right)+\sqrt{\left(\sqrt{\frac{k_{3} P_{\mathrm{C}_{6} \mathrm{H}_{12}}}{k_{1} P_{\mathrm{O}_{2}}}}+\sqrt{\frac{k_{3} P_{\mathrm{C}_{6} \mathrm{H}_{12}}}{k_{2} P_{\mathrm{O}_{2}}}}\right)^{2}+4 \frac{k_{3} P_{\mathrm{C}_{6} \mathrm{H}_{12}}}{k_{4}}}\right)^{2}}{2 \frac{k_{3} P_{\mathrm{C}_{6} \mathrm{H}_{12}}}{k_{4}}}\right)^{2}
$$

Stage scheme of oxidative dehydrogenation of methylcyclopentane into methylcyclopentene may be presented as:

$$
\begin{aligned}
& \mathrm{O}_{2}+2 \mathrm{Z}_{3} \stackrel{k_{5}}{\longrightarrow} 2 \mathrm{Z}_{3} \mathrm{O}|1| \\
& \mathrm{Z}_{3} \mathrm{O}+\mathrm{C}_{6} \mathrm{H}_{12} \stackrel{k_{6}}{\longrightarrow} \mathrm{Z}_{3} \mathrm{OC}_{6} \mathrm{H}_{12}|1| \\
& \frac{\mathrm{Z}_{3} \mathrm{OC}_{6} \mathrm{H}_{12} \stackrel{k_{7}}{\longrightarrow} \mathrm{C}_{6} \mathrm{H}_{10}+\mathrm{H}_{2} \mathrm{O}+\mathrm{Z}_{3}|1|}{\mathrm{C}_{6} \mathrm{H}_{12}+\frac{1}{2} \mathrm{O}_{2}=\mathrm{C}_{6} \mathrm{H}_{10}+\mathrm{H}_{2} \mathrm{O}}
\end{aligned}
$$

The following expression can be written for rates of the stages:

$$
r_{5}=k_{5} \theta_{5}^{2} P_{\mathrm{O}_{2}}, r_{6}=k_{6} \theta_{6} P_{\mathrm{C}_{6} \mathrm{H}_{12}}, r_{7}=k_{7} \theta_{7}
$$

In stationary conditions

$$
r=r_{5}=r_{6}=r_{7}
$$

Where $\theta_{5}, \theta_{6}, \theta_{7}$ are fractions of catalyst surface; sites with the ability adsorb atom oxygen sites with atom oxygen and sites adsorbed molecules of methylcyclopentane.

$\theta_{5}+\theta_{6}+\theta_{7}=1$

$$
\begin{aligned}
& \theta_{7}=\frac{k_{6} P_{\mathrm{C}_{6} \mathrm{H}_{12}}}{k_{7}} \theta_{6}, \quad \theta_{5}=\sqrt{\frac{k_{6} P_{\mathrm{C}_{6} \mathrm{H}_{12}} \theta_{6}}{k_{5} P_{\mathrm{O}_{2}}}}, \\
& \frac{k_{6} P_{\mathrm{C}_{6} \mathrm{H}_{12}}}{k_{7}} \theta_{6}+\theta_{6}+\sqrt{\frac{k_{6} P_{\mathrm{C}_{6} \mathrm{H}_{12}}}{k_{5} P_{\mathrm{O}_{2}}}} \sqrt{\theta_{6}}-1=0
\end{aligned}
$$

Assuming

$$
\begin{aligned}
& \frac{k_{6} P_{\mathrm{C}_{6} \mathrm{H}_{12}}}{k_{7}}+1=c, \quad \sqrt{\frac{k_{6} P_{\mathrm{C}_{6} \mathrm{H}_{12}}}{k_{5} P_{\mathrm{O}_{2}}}}=d, \quad \theta_{6}=x^{2}, \\
& \sqrt{\theta_{6}}=x
\end{aligned}
$$


we'll obtain:

$$
\begin{aligned}
& c x^{2}+d x-1=0, \\
& x=\frac{-d+\sqrt{d^{2}+4 c}}{2 c}, \\
& \theta_{6}=x^{2}=\left(\frac{-d+\sqrt{d^{2}+4 c}}{2 c}\right)^{2} .
\end{aligned}
$$

Then equation of formation rate of ethylcyclopentene appears as:

$$
r=r_{\mathrm{C}_{6} \mathrm{H}_{10}}^{1}=k_{6} P_{\mathrm{C}_{6} \mathrm{H}_{12}}\left\{\frac{-d+\sqrt{d^{2}+4 c}}{2 c}\right\}^{2}
$$

$$
r=r_{\mathrm{C}_{6} \mathrm{H}_{10}}^{1}=k_{6} P_{\mathrm{C}_{6} \mathrm{H}_{12}}\left\{\frac{-\sqrt{\frac{k_{6} P_{\mathrm{C}_{6} \mathrm{H}_{12}}}{k_{5} P_{\mathrm{O}_{2}}}}+\sqrt{\left(\sqrt{\left.\frac{k_{6} P_{\mathrm{C}_{6} \mathrm{H}_{12}}}{k_{5} P_{\mathrm{O}_{2}}}\right)^{2}+4\left(\frac{k_{6} P_{\mathrm{C}_{6} \mathrm{H}_{12}}}{k_{7}}+1\right)}\right.}}{2\left(\frac{k_{6} P_{\mathrm{C}_{6} \mathrm{H}_{12}}}{k_{7}}+1\right)}\right\}^{2}
$$

Kinetic regularities of the reaction of oxidative dehydrogenation of methylcyclopentane over metal-clinoptilolite catalyst $\mathrm{CL}-\mathrm{CuCrCoZn}$ are given in Table 1. Kinetic tests were performed in the range of temperature, $(320-360)^{0} \mathrm{C}$, at a space velocity of $(500-2000) \mathrm{h}^{-1}$, partial pressure of reagents; $P_{\mathrm{C}_{6} \mathrm{H}_{12}}=0.2196-0.3199 \mathrm{~atm}$,
Assuming that carbon dioxide is formed as a results of interaction of adsorbed molecules, methylcyclopentane, methylcylopentene and metylcyclopentadiene with adsorbed molecules of oxygen it can be written the following kinetic equations corresponding to these mechanisms.

$$
\begin{aligned}
& P_{\mathrm{O}_{2}}=0.1438-0.1659 \mathrm{~atm} \text { and } G_{\text {cat }}=1.78 \mathrm{~g}, \\
& V_{\text {cat }}=2 \mathrm{sm}^{3} .
\end{aligned}
$$

$$
\begin{aligned}
& r_{\mathrm{CO}_{2}}^{1}=\frac{k_{8} K_{1} P_{1} K_{6} P_{2}}{\left(1+K_{1} P_{1}+\sqrt{K_{2} P_{2}}+K_{3} P_{3}+K_{4} P_{4}+K_{5} P_{5}+K_{6} P_{2}\right)^{2}} \\
& r_{\mathrm{CO}_{2}}^{2}=\frac{k_{9} K_{3} P_{3} K_{6} P_{2}}{\left(1+K_{1} P_{1}+\sqrt{K_{2} P_{2}}+K_{3} P_{3}+K_{4} P_{4}+K_{5} P_{5}+K_{6} P_{2}\right)^{2}} \\
& r_{\mathrm{CO}_{2}}^{3}=\frac{k_{10} K_{4} P_{4} K_{6} P_{2}}{\left(1+K_{1} P_{1}+\sqrt{K_{2} P_{2}}+K_{3} P_{3}+K_{4} P_{4}+K_{5} P_{5}+K_{6} P_{2}\right)^{2}} \\
& r_{\mathrm{C}_{6} \mathrm{H}_{10}}=r_{\mathrm{C}_{6} \mathrm{H}_{10}}^{1}-r_{\mathrm{CO}_{2}}^{2} \\
& r_{\mathrm{C}_{6} \mathrm{H}_{8}}=r_{\mathrm{C}_{6} \mathrm{H}_{8}}^{1}-r_{\mathrm{CO}_{2}}^{3} \\
& r_{\mathrm{CO}_{2}}=r_{\mathrm{CO}_{2}}^{1}+r_{\mathrm{CO}_{2}}^{2}+r_{\mathrm{CO}_{2}}^{3}
\end{aligned}
$$


Table 1. The results of kinetic runs

\begin{tabular}{|c|c|c|c|c|c|c|c|c|c|c|c|c|c|c|}
\hline \multicolumn{2}{|c|}{$\begin{array}{l}\text { Partial pressure of } \\
\text { reagents, atm }\end{array}$} & \multirow{2}{*}{$\begin{array}{c}\begin{array}{c}\text { Moles of } \\
\text { substance, } \\
\text { mol/hr }\end{array} \\
n_{C_{6} H_{12}}^{0}\end{array}$} & \multirow{2}{*}{$\begin{array}{c}\begin{array}{c}\text { Mole of } \\
\text { substance, } \\
\mathrm{mol} / \mathrm{hr}\end{array} \\
n_{\mathrm{O}_{2}}^{0}\end{array}$} & \multirow{2}{*}{$\begin{array}{c}\begin{array}{c}\text { Mole of } \\
\text { substance, } \\
\mathrm{mol} / \mathrm{hr}\end{array} \\
n_{N_{2}}^{0}\end{array}$} & \multirow{2}{*}{$\begin{array}{c}\text { Space } \\
\text { velocity, } \\
v, \mathrm{~h}^{-1}\end{array}$} & \multirow{2}{*}{$\begin{array}{c}\text { Tempe- } \\
\text { rature, } \\
T,{ }^{0} \mathrm{C}\end{array}$} & \multirow{2}{*}{$\begin{array}{c}\text { Conver- } \\
\text { sion, } \\
X, \%\end{array}$} & \multicolumn{7}{|c|}{ Yields of products of reaction, $A, \%$} \\
\hline$P_{\mathrm{C}_{6 \mathrm{H}_{12}}}$ & $P_{\mathrm{O}_{2}}$ & & & & & & & $A_{1} \mathrm{C}_{5} \mathrm{H}_{8}$ & $A_{2} \mathrm{C}_{5} \mathrm{H}_{6}$ & $A_{3} \mathrm{C}_{6} \mathrm{H}_{10}$ & $A_{4} \mathrm{C}_{6} \mathrm{H}_{8}$ & $A_{5} \mathrm{C}_{6} \mathrm{H}_{10}$ & $A_{6} \mathrm{C}_{6} \mathrm{H}_{6}$ & $A_{7} \mathrm{CO}_{2}$ \\
\hline 0.2765 & 0.1533 & 0.0178 & 0.00987 & 0.0367 & 500 & 300 & 31.5 & - & - & 8.1 & 12.8 & 5.8 & 0.7 & 4.1 \\
\hline 0.2675 & 0.1533 & 0.0178 & 0.00987 & 0.0367 & 500 & 320 & 34.9 & 0.3 & - & 9.0 & 14.6 & 6.0 & - & 5.0 \\
\hline 0.2675 & 0.1533 & 0.0178 & 0.00987 & 0.0367 & 500 & 340 & 42.7 & 0.9 & 0.4 & 10.5 & 15.7 & 6.3 & 4.2 & 4.7 \\
\hline 0.2765 & 0.1533 & 0.0178 & 0.00987 & 0.0367 & 500 & 360 & 48.1 & 1.4 & 0.6 & 13.7 & 17.5 & 7.4 & 1.9 & 5.6 \\
\hline 0.3199 & 0.1438 & 0.0356 & 0.016 & 0.0597 & 1000 & 300 & 47.5 & 2.7 & 2.4 & 12.4 & 18.5 & 5.4 & 2.4 & 3.7 \\
\hline 0.3199 & 0.1438 & 0.0356 & 0.016 & 0.0597 & 1000 & 320 & 50.8 & 3.1 & 2.7 & 13.9 & 18.8 & 5.9 & 3.4 & 3.0 \\
\hline 0.3199 & 0.1438 & 0.0356 & 0.016 & 0.0597 & 1000 & 340 & 53.5 & 4.1 & 1.6 & 15.9 & 19.4 & 5.9 & 3.7 & 2.9 \\
\hline 0.3199 & 0.1438 & 0.0356 & 0.016 & 0.0597 & 1000 & 360 & 58 & 2.4 & 3.8 & 16.3 & 22.7 & 6.3 & 3.0 & 3.5 \\
\hline 0.2836 & 0.1512 & 0.045 & 0.024 & 0.0897 & 1500 & 300 & 54.8 & 3.8 & 2.6 & 12 & 25.9 & 2.7 & 3.5 & 4.3 \\
\hline 0.2836 & 0.1512 & 0.045 & 0.024 & 0.0897 & 1500 & 320 & 60.5 & 5.2 & 3.9 & 14.4 & 27.2 & 4.5 & 2.6 & 2.7 \\
\hline 0.2836 & 0.1512 & 0.045 & 0.024 & 0.0897 & 1500 & 340 & 70.6 & 3.1 & 4.9 & 15.8 & 36.0 & 2.3 & 4.0 & 4.5 \\
\hline 0.2836 & 0.1512 & 0.045 & 0.024 & 0.0897 & 1500 & 360 & 74.6 & 1.5 & 3.6 & 17.0 & 42.3 & 4.3 & 2.0 & 3.9 \\
\hline 0.2196 & 0.1659 & 0.045 & 0.034 & 0.126 & 2000 & 300 & 40.9 & 2.7 & 4.2 & 10.7 & 14.7 & 3.3 & 1.9 & 3.4 \\
\hline 0.2196 & 0.1659 & 0.045 & 0.034 & 0.126 & 2000 & 320 & 43.7 & 1.9 & 2.7 & 13.2 & 15.9 & 4.6 & 2.7 & 2.7 \\
\hline 0.2196 & 0.1659 & 0.045 & 0.034 & 0.126 & 2000 & 340 & 50.7 & 2.9 & 1.5 & 15.5 & 17.0 & 4.9 & 4.1 & 4.8 \\
\hline 0.2196 & 0.1659 & 0.045 & 0.034 & 0.126 & 2000 & 360 & 58.4 & 3.4 & 2.1 & 15.8 & 20.4 & 5.5 & 4.9 & 6.3 \\
\hline
\end{tabular}


Equation (8)-(10) make up the kinetic model of the considered reaction.

We can show the rate of formation of carbon dioxide from the cyclopentene, cyclopentadiene, cyclohexene, benzene, methylcyclopentene, methylcyclopentadiene and summer of methylcyclopentane, methylcyclopentene, methylcyclopentadiene, accordingly can be represented by the following differential equations:

$$
\begin{array}{ll}
\frac{\mathrm{d} A_{1}}{\mathrm{~d} \frac{G_{\mathrm{cat}}}{n_{\mathrm{C}_{6} \mathrm{H}_{12}}^{0}}}=k_{14} P_{1}, & \frac{\mathrm{d} A_{2}}{\mathrm{~d} \frac{G_{\mathrm{cat}}}{n_{\mathrm{C}_{6} \mathrm{H}_{12}}^{0}}}=k_{13} P_{6}, \\
\frac{\mathrm{d} A_{5}}{\mathrm{~d} \frac{G_{\mathrm{cat}}}{n_{\mathrm{C}_{6} \mathrm{H}_{12}}^{0}}}=k_{12} P_{1}, & \frac{\mathrm{d} A_{6}}{\mathrm{~d} \frac{G_{\mathrm{cat}}}{n_{\mathrm{C}_{6} \mathrm{H}_{12}}^{0}}}=k_{11} P_{8}
\end{array}
$$

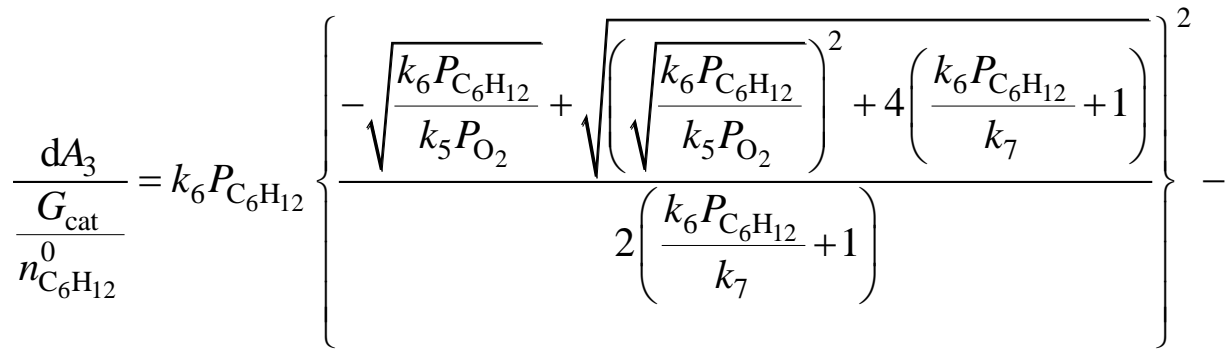

$$
\begin{aligned}
& -\frac{k_{9} K_{3} P_{3} K_{6} P}{\left(1+K_{1} P_{1}+\sqrt{K_{2} P_{2}}+K_{3} P_{3}+K_{4} P_{4}+K_{5} P_{5}+K_{6} P_{2}\right)^{2}}
\end{aligned}
$$

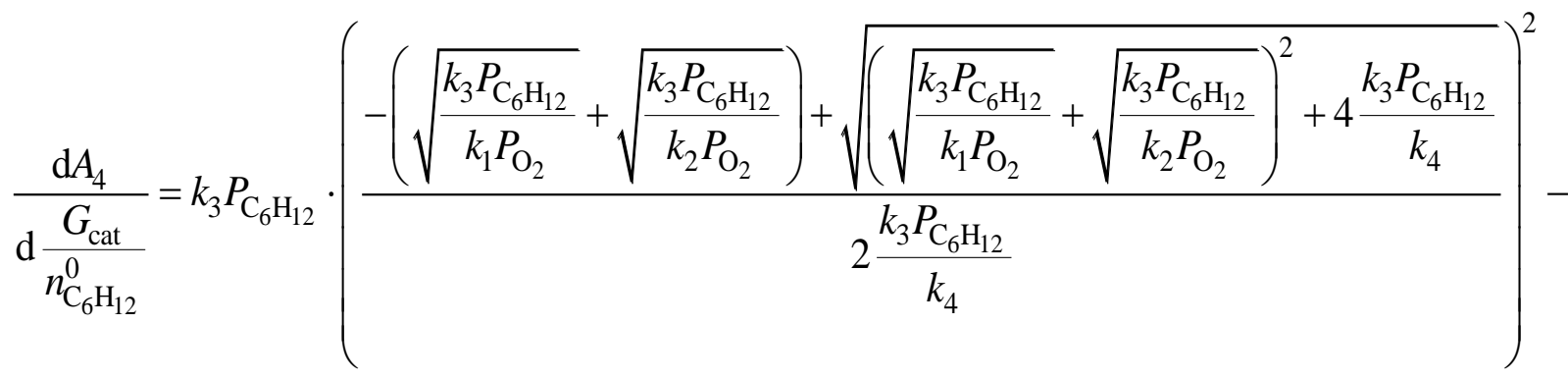

$$
\begin{aligned}
& -\frac{k_{10} K_{4} P_{4} K_{6} P_{2}}{\left(1+K_{1} P_{1}+\sqrt{K_{2} P_{2}}+K_{3} P_{3}+K_{4} P_{4}+K_{5} P_{5}+K_{6} P_{2}\right)^{2}} \\
& \frac{\mathrm{d} A_{7}}{\mathrm{~d} \frac{G_{\mathrm{cat}}}{n_{\mathrm{C}_{6} \mathrm{H}_{12}}^{0}}}=\frac{k_{8} K_{1} P_{1} K_{6} P_{2}}{\left(1+K_{1} P_{1}+\sqrt{K_{2} P_{2}}+K_{3} P_{3}+K_{4} P_{4}+K_{5} P_{5}+K_{6} P_{2}\right)^{2}}+ \\
& \frac{k_{9} K_{4} P_{4} K_{6} P_{2}}{\left(1+K_{1} P_{1}+\sqrt{K_{2} P_{2}}+K_{3} P_{3}+K_{4} P_{4}+K_{5} P_{5}+K_{6} P_{2}\right)^{2}}+\frac{k_{10} K_{3} P_{3} K_{6} P_{2}}{\left(1+K_{1} P_{1}+\sqrt{K_{2} P_{2}}+K_{3} P_{3}+K_{4} P_{4}+K_{5} P_{5}+K_{6} P_{2}\right)^{2}}
\end{aligned}
$$


here $K i$ - the adsorption equilibrium constant $\left(K_{i}=K_{i}^{0} \cdot \mathrm{e}^{\frac{Q_{i}}{R T}}\right), \quad\left(k_{i}=k_{i}^{0} \cdot \mathrm{e}^{-\frac{E}{R T}}\right) \quad k_{\mathrm{i}}-$ reaction rate constant.

The suitable stoichiometric equations of formation of the reaction products are,

1. $\mathrm{C}_{6} \mathrm{H}_{12}+\frac{1}{2} \mathrm{O}_{2}=\mathrm{C}_{6} \mathrm{H}_{10}+\mathrm{H}_{2} \mathrm{O}$

2. $\mathrm{C}_{6} \mathrm{H}_{10}+\frac{1}{2} \mathrm{O}_{2}=\mathrm{C}_{6} \mathrm{H}_{8}+\mathrm{H}_{2} \mathrm{O}$

3. $\mathrm{C}_{6} \mathrm{H}_{8}+\frac{1}{2} \mathrm{O}_{2}=\mathrm{C}_{6} \mathrm{H}_{6}+\mathrm{H}_{2} \mathrm{O}$
3. $\mathrm{C}_{6} \mathrm{H}_{12}+9 \mathrm{O}_{2}=6 \mathrm{CO}_{2}+6 \mathrm{H}_{2} \mathrm{O}$

4. $\mathrm{C}_{6} \mathrm{H}_{10}+8.5 \mathrm{O}_{2}=6 \mathrm{CO}_{2}+5 \mathrm{H}_{2} \mathrm{O}$

$$
\text { 5. } \mathrm{C}_{6} \mathrm{H}_{8}+8 \mathrm{O}_{2}=6 \mathrm{CO}_{2}+4 \mathrm{H}_{2} \mathrm{O}
$$

By using these equations, the reaction yield and the initial molar amounts of the reactants we can determine the current velocity in the molar flow of methylcyclopentane, methylcyclopentene, methylcyclopentadiene, benzene, carbon dioxide, oxygen and water accordingly by the following equations:

$$
\left.\begin{array}{l}
n_{\mathrm{C}_{6} \mathrm{H}_{12}}=n_{\mathrm{C}_{6} \mathrm{H}_{12}}^{0}-\left(A_{1} n_{\mathrm{C}_{6} \mathrm{H}_{12}}^{0}+A_{2} n_{\mathrm{C}_{6} \mathrm{H}_{12}}^{0}+A_{3} n_{\mathrm{C}_{6} \mathrm{H}_{12}}^{0}+A_{4} n_{\mathrm{C}_{6} \mathrm{H}_{12}}^{0}+A_{5} n_{\mathrm{C}_{6} \mathrm{H}_{12}}^{0}+A_{6} n_{\mathrm{C}_{6} \mathrm{H}_{12}}^{0}+A_{7} n_{\mathrm{C}_{6} \mathrm{H}_{12}}^{0}\right) / 100 \\
n_{\mathrm{C}_{5} \mathrm{H}_{8}}=A_{1} n_{\mathrm{C}_{6} \mathrm{H}_{12}} / 100 \\
n_{\mathrm{C}_{5} \mathrm{H}_{6}}=A_{2} n_{\mathrm{C}_{6} \mathrm{H}_{12}}^{0} / 100 \\
n_{\mathrm{C}_{6} \mathrm{H}_{10}}=A_{3} n_{\mathrm{C}_{6} \mathrm{H}_{12}}^{0} / 100 \\
n_{\mathrm{C}_{6} \mathrm{H}_{8}}=A_{4} n_{\mathrm{C}_{6} \mathrm{H}_{12}}^{0} / 100 \\
n_{\mathrm{C}_{6} \mathrm{H}_{10}}=A_{5} n_{\mathrm{C}_{6} \mathrm{H}_{12}}^{0} / 100 \\
n_{\mathrm{C}_{6} \mathrm{H}_{6}}=A_{6} n_{\mathrm{C}_{6} \mathrm{H}_{12}}^{0} / 100 \\
n_{\mathrm{CO}_{2}}=6 A_{7} n_{\mathrm{C}_{6} \mathrm{H}_{12}}^{0} / 100 \\
n_{\mathrm{O}_{2}}=n_{\mathrm{O}_{2}}^{0}-\left(\frac{1}{2} A_{3} n_{\mathrm{C}_{6} \mathrm{H}_{12}}^{0}+\frac{1}{2} A_{4} n_{\mathrm{C}_{6} \mathrm{H}_{12}}^{0}+8.5 A_{7} n_{\mathrm{C}_{6} \mathrm{H}_{12}}^{0}\right) / 100 \\
n_{\mathrm{H}_{2} \mathrm{O}}=\left(A_{1} n_{\mathrm{C}_{6} \mathrm{H}_{12}}^{0}+A_{2} n_{\mathrm{C}_{6} \mathrm{H}_{12}}^{0}+A_{3} n_{\mathrm{C}_{6} \mathrm{H}_{12}}^{0}+A_{4} n_{\mathrm{C}_{6} \mathrm{H}_{12}}^{0}+A_{5} n_{\mathrm{C}_{6} \mathrm{H}_{12}}^{0}+A_{6} n_{\mathrm{C}_{6} \mathrm{H}_{12}}^{0}+5 A_{7} n_{\mathrm{C}_{6} \mathrm{H}_{12}}^{0}\right) / 100 \\
n_{\mathrm{N}_{2}}=n_{\mathrm{N}_{2}}^{0}
\end{array}\right\}
$$

The partial pressure of the reactants expressed by the equation:

$$
P_{i}=\frac{n_{i} p}{\sum n_{i}}
$$

$A_{1}, A_{2}, A_{3}, A_{4}, A_{5}, A_{6}, A_{7}-$ yields of cyclopentene, cyclopentadiene, methylcyclopentene, methylcyclopentadiene, cyclohexene, benzene and carbon dioxide respectively. $P_{1}, P_{2}$, $P_{3}, P_{4}, P_{5}, P_{6}, P_{7}, P_{8} P_{9}-$ partial pressure of methylcyclopentane, oxygen, methylcyclopentene, methylcyclopentadiene, benzene, cyclopentene, cyclopentadiene, cyclohexene and water accordingly.

Equations (4) and (6), (8) (10) form the kinetic model of the process.

A kinetic model of the reaction subjected to statistical analysis on the basis of kinetic data. Calculation of pre-exponential factors of the reaction constants, activation energies and heats of adsorption conducted by methods of "rolling admission" and Pauelusing software system "Search" [7], where the objective function has the form:

$$
F=\min \sum_{j=l}^{m} \sum_{i=l}^{n}\left\{\frac{A_{j i}^{\mathrm{exp}}-A_{j i}^{\mathrm{calc}}}{A_{j i}^{\exp }}\right\}
$$

$A_{j i}^{\mathrm{exp}}, A_{j i}^{\mathrm{calc}}-$ the experimental and calculated values of outputs $i$-th component in the $j$ experiment, $m$ - the number of experiments, $n-$ the number of components.

Numerical values of the constants of a kinetic model presented in the Table 2. Calculations showed that the relative error of experimental and calculated data did not exceed $10 \%-15 \%$. 
Table 2. The kinetic parameters of kinetic models

\begin{tabular}{|c|c|c|c|}
\hline \multicolumn{3}{|c|}{ The kinetic model of reaction oxidative dehydrogenation of methylcyclopentane } \\
\hline \multicolumn{2}{|c|}{$\ln k_{1}^{0}\left(\ln K_{1}^{0}\right)$} & $E_{1}$ & $E_{i}\left(Q_{i}\right), \mathrm{kcal} /$ mole \\
\hline $\ln k_{1}^{0}$ & 30.34 & $E_{2}$ & 69.98 \\
\hline $\ln k_{2}^{0}$ & 13.81 & $E_{3}$ & 93.06 \\
\hline $\ln k_{3}^{0}$ & 21.68 & $E_{4}$ & 51.95 \\
\hline $\ln k_{4}^{0}$ & 31.22 & $E_{5}$ & 30.11 \\
\hline $\ln k_{5}^{0}$ & 15.68 & $E_{6}$ & 32.14 \\
\hline $\ln k_{6}^{0}$ & 27.14 & $E_{7}$ & 52.40 \\
\hline $\ln k_{7}^{0}$ & 29.27 & $E_{8}$ & 37.93 \\
\hline $\ln k_{8}^{0}$ & -17.23 & $E_{9}$ & 27.34 \\
\hline $\ln k_{9}^{0}$ & -15.82 & $E_{10}$ & 89.80 \\
\hline $\ln k_{10}^{0}$ & 69.78 & $E_{11}$ & 30.04 \\
\hline $\ln k_{11}^{0}$ & 10.78 & $E_{12}$ & 15.02 \\
\hline $\ln k_{12}^{0}$ & 94.12 & $E_{13}$ & 15.17 \\
\hline $\ln k_{13}^{0}$ & 14.40 & $E_{14}$ & 19.87 \\
\hline $\ln k_{14}^{0}$ & 82.98 & $Q_{1}$ & 15.00 \\
\hline $\ln K_{1}^{0}$ & 57.41 & $Q_{2}$ & 98.19 \\
\hline $\ln K_{2}^{0}$ & 60.38 & $Q_{3}$ & 37.10 \\
\hline $\ln K_{3}^{0}$ & 41.89 & $Q_{4}$ & 47.50 \\
\hline $\ln K_{4}^{0}$ & 12.46 & $Q_{5}$ & 90.32 \\
\hline $\ln K_{5}^{0}$ & 50.35 & $Q_{1}$ & 28.54 \\
\hline $\ln K_{6}^{0}$ & 23.36 & & 81.11 \\
\hline
\end{tabular}

\section{References}

1. Pines H., Csicery S.M. Dehydrogenation, Dehydrocyclization and Isomerization of $\mathrm{C}_{5}-\mathrm{C}_{6}$ Hydrocarbons over Chromia-Alumina Catalysts. J. American Chem. Soc. 1962. V. 84. P. 292. http:// dx.doi.org/10.1021/ja00861a032

2. Agadadash M.Aliyev, Zumrud A.Shabanova, Ulvi M. Najaf-Guliyev, Irada G. Melikova. Selection of Active Modified Zeolite Catalyst and Kinetics of the Reaction of Selective Oxidative Dehydrogenation of Cyclohexane to Cyclohexadiene 1,3. Mo-dern Research in Catalysis. 2015. V. 4. P. 8796. http://dx.doi.org/10.4236/mrc.2015.44011

3. Tagiyev D.B., Minachev H.M. Catalytic Properties of Zeolites in Oxidation Reaction. Uspekhi khimii. 1981. V. 50. P. 1929-1259.
4. Process and catalyst for the preparation of methyl1,3-cyclopentadiene. Clarence L. Dulaney, Texas City, Tex., Raymond A.Franz, Kirkwood, Mio, assignors to Monsanto Company, St. Louis, Mo., a corporation of Delaware No Drawing. Filed Mar. 8, 1962. Ser. No. 178. Claims. P. 260-666.

5. Zhao D., Cleare K., Oliver C., Ingram C., Cook D., Szostak R., Kevan L. "Characteristics of the synthetic heulandite-clinoptilolite family of zeolites", Microporous and Mesoporous Materials. 1998. V. 21. P. 371-379.

6. Gottardi G., Galli E. Natural Zeolites. SpringerVerlag, Berlin, 1985.

7. Shakhtakhtinsky T.N., Bakhmanov M.F., Kelbaliyev G.N. Methods of Optimization of Processes of Chemical Engineering with the Computer Programs. Baku: Elm, 1985. P. 260.

\section{METILTSIKLOPENTANIN SELEKTIV OKSIDLOŞDIRICI DEHIDROGENLəŞMOSI REAKSIYASININ MEXANIZIMİ Və KINNETIKASI}

\section{A.M.Đliyev, M.Y.Abbasov, M.Q.Đliyeva, G.Ө.Đlizadə, R.Y.Ağayeva}

Alitsiklik dien karbohidrogenlərinin oksigenin iştirakı ilə gedən oksidləşdirici dehidrogenləşməsi az öyrənilmiş heterogen katalitik reaksiyalara aiddir. Metilsiklopentanın dehidrogenləşməsi yüksək dərəcədə gedən endotermik reaksiyadır. Reaksiyanın kinetikasını üçün, bu tədqiqatda məqsəd əlaqəli bir katalitik təbəqə saxlaya bilən keçirici metalların ( $\mathrm{Cu}, \mathrm{Zn}, \mathrm{Co}, \mathrm{Cr})$ köməyi ilə strukturlaşdırılmış bir katalizator hazırlamaq idi. Katalizatorun aktiv fazasında keçid metalları adsorbsiya edilərək metiltsiklopentanın dehdirogenləşməsi reaksiyasında test edildi. Katalizatorun hazırlanması mərhələsi oksidləşmə reaksiyasının aparılması üçün uyğun olan üç əsas mərhələni əhatə edir: aktiv hissəciklərin katalizator səthinə yüklənməsi, səthdə aktiv kataliaztor təbəqəsinin hazırlanmsı və nəhayət katalizatorun aktiv fazaya gətirilməsi. Bu araşdırmada müxtəlif növ katalizatorların aktivasiyası və deaktivasiyası mexanizmlərinin stabilliyi öyrənilmişdir. Bu işin üstünlüyü metiltsiklopentanın oksidləşdirici dehidrogenləşməsinin havanın oksigeni 
hesabına baş verməsidir. Seolitin quruluşunun öyrənilməsi katalizator kombinasiyalarının müəyyən etməyə və metalların klinoptilolit üzərində adsorbsiyasına və katalizator hissəciklərinin dispers seçiciliyinə köməklik göstərdi. Kinetik parametrlərin ədədi qiymətləri hesablandı.

Açar sözlor: kinetika, oksidlaşdirici dehidrogenlaşmə, metilsiklopentadien-1,3.

\section{МЕХАНИЗМ И КИНЕТИКА РЕАКЦИИ СЕЛЕКТИВНОГО ОКИСЛИТЕЛЬНОГО ДЕГИДРИРОВАНИЯ МЕТИЛЦИКЛОПЕНТАНА}

\section{А.М.Алиев, М.Я.Аббасов, М.К.Алиева, Г.А.Ализаде, Р.Ю.Агаева}

Окислительное дегидрирование алициклических диеновых углеводородов в присутствии кислорода является малоизученной гетерогенно-каталитической реакцией. Дегидрирование метилциклопентена - эндотермическая реакция. Для изучения кинетики реакции, разработан структурированный катализатор с катионами металлов $(\mathrm{Cu}, \mathrm{Zn}, \mathrm{Co}, \mathrm{Cr})$, которые связаны с поверхностью катализатора. В активной фазе катализатора переходные металлы, были адсорбированы и протестированы реакцией дегидрирования метилциклопентена. Фаза приготовления катализатора охватывает три основных стадии, соответствующие реакциям окисления: нанесение активных частиц на поверхность катализатора, приготовление активного слоя катализатора на поверхности и, наконец, перевод катализатора в активную фазу. Изучена устойчивость активации и дезактивации механизмов различных типов катализаторов. Преимущество данной работы состоит в том, что окислительное дегидрирование метилциклопентена происходит за счет кислорода воздуха. Исследование структуры цеолита помогло определить комбинации катализаторов и адсорбцию металлов на клиноптилолите, а также селективность диспергирования частиц катализатора. Рассчитаны численные значения кинетических параметров.

Ключевые слова: кинетика, окислительное дегидрирование, метилциклопентадиен-1,3. 